\title{
Sirtuin-6 Preserves R-spondin-1 Expression and Increases Resistance of Intestinal Epithelium to Injury in Mice
}

\author{
Fangyi Liu, ${ }^{1,2,3}$ Heng-Fu Bu, ${ }^{2,3}$ Hua Geng, ${ }^{2,3}$ Isabelle G De Plaen, ${ }^{2,3}$ Chao Gao, ${ }^{4}$ Peng Wang, ${ }^{2,3}$ Xiao Wang, ${ }^{2,3}$ \\ Jacob A Kurowski, ${ }^{2,3}$ Hong Yang, ${ }^{1}$ Jiaming Qian, ${ }^{1}$ and Xiao-Di Tan ${ }^{2,3,5}$
}

${ }^{1}$ Department of Gastroenterology, Peking Union Medical College Hospital, Chinese Academy of Medical Sciences and Peking Union Medical College, Beijing, People's Republic of China; ${ }^{2}$ Center for Intestinal and Liver Inflammation Research, Stanley Manne Children's Research Institute, Ann and Robert H Lurie Children's Hospital of Chicago, Chicago, Illinois, United States of America; ${ }^{3}$ Department of Pediatrics, Feinberg School of Medicine, Northwestern University, Chicago, Illinois, United States of America; ${ }^{4}$ Center of Clinical Reproductive Medicine, State Key Laboratory of Reproductive Medicine, The First Affiliated Hospital of Nanjing Medical University, Nanjing, Jiangsu, People's Republic of China; and ${ }^{5}$ Department of Research and Development, Jesse Brown VA Medical Center, Chicago, Illinois, United States of America

\begin{abstract}
Sirtuin-6 (Sirt6) is a critical epigenetic regulator, but its function in the gut is unknown. Here, we studied the role of intestinal epithelial Sirt6 in colitis-associated intestinal epithelial injury. We found that Sirt6, which is predominantly expressed in epithelial cells in intestinal crypts, is decreased in colitis in both mice and humans. Colitis-derived inflammatory mediators including interferon- $\gamma$ and reactive oxygen species strongly inhibited Sirt6 protein expression in young adult mouse colonocyte (YAMC) cells. The susceptibility of the cells to injurious insults was increased after knockdown of Sirt6 expression. In contrast, YAMC cells with Sirt6 overexpression exhibited more resistance to injurious insult. Furthermore, intestinal epithelial-specific Sirt6 (Sirt6 ${ }^{I E C-k O}$ ) knockout mice exhibited greater susceptibility to dextran sulfate sodium (DSS)-induced colitis. RNA sequencing transcriptome analysis revealed that inflammatory mediators such as tumor necrosis factor (TNF)- $\alpha$ suppressed expression of R-spondin-1 (Rspol, a critical growth factor for intestinal epithelial cells) in Sirt6-silenced YAMC cells in vitro. In addition, lipopolysaccharide was found to inhibit coIonic Rspol expression in Sirt6 ${ }^{\text {ECCKO }}$ mice but not their control littermates. Furthermore, Sirt6 ${ }^{I E C-K O}$ mice with DSS-induced colitis also exhibited in a significant decrease in Rspol expression in colons. In vitro, knockdown of Rspol attenuated the effect of ectopic expression of Sirt6 on protection of YAMC cells against cell death challenges. In conclusion, Sirt6 plays an important role in protecting intestinal epithelial cells against inflammatory injury in a mechanism associated with preserving Rspol levels in the cells.
\end{abstract}

Online address: http://www.molmed.org

doi: $10.2119 /$ molmed.2017.00085

\section{INTRODUCTION}

Inflammatory bowel disease (IBD) is an idiopathic disorder affecting approximately 1.5 million Americans and others worldwide $(1,2)$. The incidence of IBD seems to be increasing in many regions of the world $(2,3)$. Crypt epithelial cell damage associated with colonic inflammation is a distinctive pathological hallmark of IBD (4). However, prevention and treatment of IBD remain a challenge, as the pathogenesis of the
Address correspondence to Xiao-Di Tan, Stanley Manne Children's Research Institute Ann and Robert H Lurie Children's Hospital of Chicago, 225 E. Chicago Avenue, Box 217, Chicago, IL 60611 , USA. Phone: (773) 755-6380; Fax: (773) 755-6581; E-mail: xtan@northwestern.edu. Or to Jiaming Qian, Department of Gastroenterology, Peking Union Medical College Hospital, No. 1, Shuaifuyuan, Dongcheng District, Beijing, 100730, People's Republic of China. Tel: (86) $1069155019 ;$

E-mail: qianjiaming1957@126.com

Submitted May 17, 2017; Accepted for Publication August 28, 2017;

Published Online (www.molmed.org) October 23, 2017.

$\exists$

Feinstein Institute

for Medical Research Northwell Health disease is unknown (5). Therefore, there is a strong rationale for exploring novel therapeutic targets that can be applied to strengthen epithelial cells against injurious insults for management of patients with IBD.

The sirtuins are a family of proteins that mainly function as nicotinamide adenine dinucleotide-dependent deacetylases (6). In mammals, there are seven sirtuin proteins, Sirt1 to Sirt7 (6). Among them, Sirt1 and Sirt6 are the most well-characterized, with functions associated with inflammation. Sirt1 is a cytosolic protein with nuclear translocation ability. Previous studies have shown that Sirt1 plays a role in regulation of inflammatory signals in the gut $(7,8)$. In contrast, Sirt6 is a nuclear and chromatin-bound protein that emerges as an important epigenetic regulator 
controlling a number of metabolic and survival processes in cells (9). Recently, Kanfi et al. reported that mice overexpressing Sirt6 have increased longevity (10). Sirt6 knockout mice were found to die within one month after birth due to numerous abnormalities, including profound lymphopenia, loss of subcutaneous fat, lordokyphosis and severe metabolic defects (11). Evidence suggests that Sirt6 has both proinflammatory and antiinflammatory roles, depending on the context and cell type involved (9). Currently, the exact role of Sirt6 in intestinal epithelial cells remains unknown, and how it is affected during intestinal inflammation has not been explored.

In this study, our objectives were to evaluate the function of Sirt6 in intestinal epithelial cells and to determine the link between Sirt6 and colitis-associated intestinal epithelial injury using both in vivo and in vitro approaches. To this end, we first studied whether colitis development was associated with alterations in colonic Sirt6 expression in mice and humans. Then, using mice with intestinal epithelial cells deficient in the Sirt6 gene, we assessed the role of epithelial cell Sirt6 in dextran sulfate sodium (DSS)-induced intestinal injury. Furthermore, we confirmed the direct role of Sirt6 in intestinal epithelial cells in vitro in response to specific injurious insults using gain- and loss-of-function approaches. Finally, we explored downstream molecules mediating the protective effects of Sirt6 on intestinal epithelial cell damage induced by inflammation using gene silencing and RNA sequencing (RNA-seq) technology. We found that Sirt6 plays an important role in maintaining intestinal epithelial cell resistance to injurious insult via a mechanism involving R-spondin-1 (Rspo1) protein, a critical epithelial mitogen that stimulates intestinal crypt cell growth in inflammation. This study may open a new avenue for the development of therapeutic targets to maintain intestinal epithelial integrity in colitis in the future.

\section{MATERIALS AND METHODS}

\section{Animals}

C57BL/6J mice, Villin-Cre transgenic mice and Sirt6 conditionally mutated $\left(\right.$ Sirt6 $\left.^{\mathrm{C} \circ / \mathrm{C} \circ}\right)$ mice were obtained from Jackson Laboratories (Bar Harbor, ME, USA). The Villin-Cre transgenic mice express Cre recombinase specifically in intestinal epithelium (12), whereas the Sirt $6^{\mathrm{C} o / \mathrm{C}}$ mice possess loxP sites flanking exons 2-3 of the Sirt6 gene, as previously described (13). Intestinal epitheliumspecific Sirt6 knockout mice (Sirt6 ${ }^{\mathrm{Co} / \mathrm{Co}}$, Villin-Cre or Sirt6 ${ }^{I E C-K O}$ mice) were generated by intercrossing Sirt $6^{\mathrm{Co} / \mathrm{C}_{0}}$ mice with Villin-Cre mice. Mouse genotypes were confirmed using polymerase chain reaction (PCR)-based protocols provided by Jackson Laboratories. Co-housed littermates carrying the LoxP-flanked alleles but not expressing Cre recombinase were used as controls for Sirt6 $6^{I E C-K O}$ mice. All mice were housed in a specific pathogen-free animal facility at the Stanley Manne Children's Research Institute. Animal husbandry was provided by trained technicians and veterinarians.

All the animal experimental procedures were conducted in accordance with the National Institutes of Health guidelines and were approved by the Institutional Animal Care and Use Committee of Northwestern University.

\section{Induction of Colitis}

Colitis was induced in mice using our previously published protocol (14). Briefly, mice (male, 8-10 wks old) fed a normal diet were given ad libitum access to 3.5\% DSS (m.w. 36,000-50,000; MP Biomedicals, Solon, OH, USA) to drink for up to $1 \mathrm{wk}$. Control mice were given regular drinking water. During experiments, mice were weighed and stools were tested for occult blood on a daily basis. The disease activity index of clinical colitis was calculated using our well-established scoring system, as previously described (14). At the end of time points indicated, mice were euthanized by $\mathrm{CO}_{2}$ inhalation. Colons and blood were collected. Blood was processed for isolation of plasma by centrifugation at $1,000 \mathrm{~g}$ for $10 \mathrm{~min}$ at $4^{\circ} \mathrm{C}$. Plasma samples and colonic tissues were stored at $-80^{\circ} \mathrm{C}$ until used. In some experiments, whole colon tissues were processed for histological analysis, as described below.

\section{Histology and Microscopic Examination}

The fresh colonic tissues were fixed in $10 \%$ neutral buffered formalin and processed for routine histology as described before (15). Hematoxylin and eosin stained sections $(5 \mu \mathrm{m})$ were examined under a light microscope using a scoring system in a blinded manner (14). Briefly, three independent parameter scores were measured: severity of inflammation (0 to 3: none, slight, moderate, severe); depth of injury ( 0 to 3: none, mucosal, mucosal and submucosal, transmural); and crypt damage (0 to 4: none, basal one-third damaged, basal two-thirds damaged, only surface epithelium intact, entire crypt and epithelium lost). Then, percent involvement of the colon tissues was estimated. Each parameter score was multiplied by the percentage of tissue involved and totals were added together to obtain a histopathology score.

\section{Immunofluorescent Staining}

Deparaffinized slides were processed for immunofluorescent staining using a method modified from our previously published protocol (16). Specifically, deparaffinized slides were hydrated through series alcohol, incubated in $10 \mathrm{mM}$ sodium citrate buffer $(\mathrm{pH}$ 6.0) at $95-100^{\circ} \mathrm{C}$ for $15 \mathrm{~min}$ for antigen retrieval, and gradually cooled to room temperature for $45 \mathrm{~min}$. Then, slides were treated with $10 \%$ goat serum (Vector Labs, Burlingame, CA, USA) for $1 \mathrm{~h}$ at room temperature to block nonspecific binding sites in tissues. Thereafter, slides were stained with rabbit monoclonal antibody against Sirt6 (1:50, CST, Danvers, MA, USA) at $4^{\circ} \mathrm{C}$ overnight. The negative control slides were stained with naïve rabbit IgG (R\&D, Minneapolis, 
MN, USA). After washing with phosphate buffered saline (PBS), slides were incubated with goat anti-rabbit IgG antibody labeled with Alexa Fluor 488 (1:250; Thermo Fisher Scientific, Waltham, MA, USA) for $90 \mathrm{~min}$ at $37^{\circ} \mathrm{C}$ in the dark. Finally, slides were washed with PBS and mounted using mounting solution containing DAPI (Vector Labs). Slides were examined under an upright fluorescence microscope (model MD R; Leica Microsystems) using appropriate filters. Images were acquired with a digital camera (QImaging Retiga 4000R), transferred to an Apple iMac computer, analyzed by Openlab image analysis software and assembled with Adobe Photoshop CS5 software.

\section{Human Samples}

All studies were performed in accordance with protocols approved by the Human Research Ethics Committee at Peking Union Medical College Hospital, Beijing, China. In this study, we recruited 15 patients with ulcerative colitis (UC) who required diagnostic colonoscopy and 17 healthy individuals undergoing colonoscopy for colorectal cancer screening at the Department of Gastroenterology at Peking Union Medical College Hospital. Under diagnostic colonoscopy procedures, two colonic biopsies were taken from sites showing active mucosal inflammation in the sigmoid colon in patients with UC. Comparable noninflamed sites of colonic mucosa in individuals who were not subsequently diagnosed with inflammatory injury in their colons were used as controls. Immediately after biopsy, a portion of samples was processed for routine histology and pathologic assessment, and the rest was immersed in RNAlater solution (Thermo Fisher Scientific) and stored at $-80^{\circ}$ for RNA extraction and quantitative reverse transcription (RT)-PCR.

\section{Cell Cultures}

Conditionally immortalized young adult mouse colonocyte (YAMC) and HT-29 cell lines were used. YAMC cells (passage 7-15) were used according to the protocol described by Whitehead et al. (17). Briefly, the cells were maintained in RPMI 1640 medium (Thermo Fisher Scientific) containing 5\% heatinactivated fetal bovine serum (Thermo Fisher Scientific), $5 \mathrm{U} / \mathrm{mL}$ mouse interferon- $\gamma$ (IFN- $\gamma$; PeproTech, Rocky Hill, NJ, USA), 100 unit/mL penicillin, 100 $\mu \mathrm{g} / \mathrm{mL}$ streptomycin (Thermo Fisher Scientific), $5 \mu \mathrm{g} / \mathrm{mL}$ insulin, $5 \mu \mathrm{g} / \mathrm{mL}$ transferrin and $5 \mathrm{ng} / \mathrm{mL}$ selenous acid (\#354351; BD Biosciences, San Jose, CA, USA) at $33^{\circ} \mathrm{C}$ as permissive conditions in a water-saturated atmosphere incubator with $5 \% \mathrm{CO}_{2}$ (18). Before experiments, the medium was replaced with one without IFN- $\gamma$ and the cells were moved to $37^{\circ} \mathrm{C}$ (a nonpermissive temperature) for $24 \mathrm{~h}$ to allow them to differentiate. Then, cells were continuously cultured under nonpermissive conditions (IFN- $\gamma$-free and $37^{\circ} \mathrm{C}$ ) for the duration of the experiment. HT-29 cells (a human intestinal epithelial cell line derived from colonic adenocarcinoma) were purchased from American Type Culture Collection (ATCC, Rockville, MD, USA) and cultured in a water-saturated atmosphere with $5 \% \mathrm{CO}_{2}$ at $37^{\circ} \mathrm{C}$. HT-29 cells (passages $20-35$ after receipt from ATCC) were maintained in Dulbecco's Modified Eagle Minimum Essential Medium (Thermo Fisher Scientific) containing $100 \mathrm{unit} / \mathrm{mL}$ penicillin, $100 \mu \mathrm{g} / \mathrm{mL}$ streptomycin and $10 \%$ heat-inactivated fetal bovine serum.

\section{Preparation of Plasmid Constructs}

A PCR fragment encoding the full open reading frame of Sirt6 was generated from mouse mRNA by RT-PCR and processed for DNA sequencing. The sequence data were compared against the National Center for Biotechnology Information database using BLAST. The mouse Sirt6 coding DNA sequence with no mutations and mismatches was inserted into XhoI and NheI sites of pIRES2-zsGreen1 expression plasmid using a standard molecular cloning protocol of our lab (19). The construct was sequenced to verify the correct orientation and sequence of the insert and was named pIRES2zsGreen1-mSirt6.

\section{Cell Transfections}

YAMC cells were seeded onto 6-well plates at densities ranging from 1.5 to $3 \times 10^{5}$ cells per well and cultured with complete medium at $33^{\circ} \mathrm{C}$ overnight. On the second day, the cells were transfected with plasmid DNA ( $2 \mu \mathrm{g} /$ well) or nucleic acids of siRNAs ( $50 \mathrm{nM}$ ) by using lipofectamine 2000 (Thermo Fisher Scientific) according to the manufacturer's protocol. After $24 \mathrm{~h}$, the medium was replaced with interferon- $\gamma$-free culture medium. Cells were then cultured at $37^{\circ} \mathrm{C}$ for an additional $24 \mathrm{~h}$ and processed for experiments or harvested for molecular biology studies. siRNAs of Sirt6, Rspo1 or their controls with unrelated sequences were purchased from Origene (Rockville, MD, USA).

\section{RNA Extraction}

Total RNA was isolated from tissues and cultured cells using Trizol solution (Thermo Fisher Scientific), as recommended by the manufacturer. To avoid contamination by DSS, which has been reported to inhibit the activity of both polymerase and reverse transcriptase (20), RNA extracted from colons of DSStreated mice were further purified using a lithium chloride method previously described by Viennois et al. (20). The concentration of RNA was determined by absorbance at $260 \mathrm{~nm}$, and the purity was checked by the $260: 280 \mathrm{~nm}$ ratio (between 1.8 and 2.0) using an ultraviolet spectrophotometer.

\section{Quantitative Real-Time RT-PCR}

Single-stranded cDNA $(20 \mu \mathrm{L})$ was generated from total RNA samples using iScript cDNA synthesis kit (Bio-Rad, Hercules, CA, USA) according to the protocol provided by the manufacturer, and diluted to $80 \mu \mathrm{L}$ with $\mathrm{ddH}_{2} \mathrm{O}$. Quantitative real-time PCR for measuring transcripts of a target gene was performed using a protocol modified from our previously published methods (14). Briefly, 96-well microtiter plates were 
used for PCR reactions. Each well contained $7.5 \mu \mathrm{L} 2 \times$ SYBR Green PCR Universal Mastermix (Applied Biosystems, Foster City, CA, USA), $0.5 \mu \mathrm{L}$ of $10 \mu \mathrm{M}$ forward primer, $0.5 \mu \mathrm{L}$ of $10 \mu \mathrm{M}$ reverse primer, $2.5 \mu \mathrm{L}$ of $\mathrm{ddH}_{2} \mathrm{O}$ and $4 \mu \mathrm{L}$ of diluted single-stranded cDNA. PCR reactions were conducted in duplicate for each sample using the Fast 7500 real-time PCR system (Applied Biosystems) under the following conditions: $50^{\circ} \mathrm{C}$ for $5 \mathrm{~min}$, $95^{\circ} \mathrm{C}$ for $10 \mathrm{~min}$, and then 40 cycles of amplification $\left(95^{\circ} \mathrm{C}\right.$ for $15 \mathrm{sec}$ and $60^{\circ} \mathrm{C}$ for $1 \mathrm{~min}$ ). The cycle at which each sample crossed a fluorescence threshold, CT (at 0.1-0.2 fluorescence units), was determined. The duplicate values for each cDNA were averaged. Fold changes in expression levels of mRNA of a target gene in samples were calculated by using the $2^{-\Delta \Delta \mathrm{CT}}$ method by using GAPDH transcripts as the internal reference (21). The $\Delta \Delta \mathrm{CT}$ value is defined as the CT difference between the normalized amount of sample and the normalized amount of calibrator. Sequences of forward (F) and reverse (R) primers for real-time PCR are listed in Table 1.

\section{Protein Extraction and Western Blot}

Total proteins were isolated from tissues and cells using our previously described protocol (22). The protein concentrations were measured using a Pierce BCA protein assay kit (Thermo Fisher Scientific), following the protocol provided by the manufacturer. Then, proteins (10 $\mu \mathrm{g} /$ sample) were mixed with $2 \times$ Laemmli sample buffer (Bio-Rad) and boiled for $5 \mathrm{~min}$ for denaturing. The denatured total proteins were resolved on 4-20\% TGX precast sodium dodecyl sulfate polyacrylamide gel electrophoresis gels (Bio-Rad) and transferred onto polyvinylidene difluoride membranes (Bio-Rad) as previously described (22). The membranes containing sample proteins were used for immunodetection of Sirt6 or Rspo1 proteins. Briefly, blots preincubated with PBS containing 5\% nonfat dry milk (Bio-Rad) were incubated with primary Ab against Sirt6 (1:1000, CST) or Rspo1 (1:200, LsBio, Seattle, WA, USA) with gentle shaking at $4^{\circ} \mathrm{C}$ overnight. After incubation, blots were washed three times with PBS containing 0.1\%

Table 1. Sequence of qRT-PCR primers

\begin{tabular}{|c|c|}
\hline mouse Sirt6-F & 5'-AGGCCGTCTGGTCATTGTC-3' \\
\hline mouse Sirt6-R & 5'-GCACATCACCTCATCCACGTA-3' \\
\hline mouse Rspo 1-F & 5'-CGACATGAACAAATGCATCA-3' \\
\hline mouse Rspo l-R & 5'-CTCCTGACACTTGGTGCAGA-3' \\
\hline mouse Gapdh-F & 5'-AACTTTGGCATTGTGGAAGG-3' \\
\hline mouse Gapdh-R & 5'-ACACATTGGGGGTAGGAACA-3' \\
\hline human SIRT6-F & 5'-CCCGGATCAACGGCTCTATC-3' \\
\hline human SIRT6-R & 5'-GCCTTCACCCTITTGGGGG-3' \\
\hline human GAPDH-F & 5'-TGCACCACCAACTGCTTAGC-3' \\
\hline human GAPDH-R & 5'-GGCATGGACTGTGGTCATGAG-3' \\
\hline mouse Tnf- $\alpha-F$ & 5'-CCACCACGCTCTTCTGTCTA-3' \\
\hline mouse Tnf- $\alpha-R$ & 5'-AGGGTCTGGGCCATAGAACT-3' \\
\hline mouse Ifn- $\gamma-F$ & 5'-TCAAGTGGCATAGATGTGGAAGAA-3' \\
\hline mouse Ifn- $\gamma-R$ & 5'-TGGCTCTGCAGGATTTTCATG-3' \\
\hline mouse $116-\mathrm{F}$ & 5'-ACCAGAGGAAATTTTCAATAGGC-3' \\
\hline mouse $\| 6-R$ & 5'-TGATGCACTTGCAGAAAACA-3' \\
\hline mouse $/ 110-\mathrm{F}$ & 5'-TGAATTCCCTGGGTGAGAAG-3' \\
\hline mouse $/ 110-R$ & 5'-TGGCCTTGTAGACACCTTGG-3' \\
\hline mouse $M c p-7-F$ & 5'-AGGTCCCTGTCATGCITCTG-3' \\
\hline mouse Mcp-7-R & 5'-TCTGGACCCATTCCTTCTTG-3' \\
\hline mouse Mip-2 $\alpha-F$ & 5'-GGGAGAGGGTGAGTTGGG-3' \\
\hline mouse Mip-2 $\alpha-R$ & 5'-GCACACTCCTTCCATGAAAGC-3' \\
\hline mouse $K c-F$ & 5'-AAAAGGTGTCCCCAAGTA-3' \\
\hline mouse $K c-R$ & 5'-AAGCAGAACTGAACTACCATCG-3' \\
\hline
\end{tabular}

Tween 20, and then incubated with 5\% nonfat dry milk containing 1/6000 diluted horseradish peroxide-conjugated goat anti-rabbit antibody (Jackson ImmunoResearch, West Grove, PA, USA) for $2 \mathrm{~h}$ at room temperature. After additional washing with PBS containing $0.1 \%$ Tween 20 , immune complexes on the blot were developed with an ECL kit (Thermo Fisher Scientific) and visualized by Bio-Rad ChemiDoc MP System (Bio-Rad). Images were analyzed with Imaging Lab 4.1 software (Bio-Rad). For detection of housekeeping gene expression, blots were stripped and reprobed with a horseradish peroxide-conjugated mouse mAb against $\beta$-actin (1:50,000, clone AC-15, Sigma-Aldrich, St. Louis, MO, USA) followed by development with the ECL kit, scanning and analyzing as described above.

\section{Induction of Cell Death and Assessment of Cell Viability}

It has been shown that development of IBD is associated with numerous types of intestinal epithelial cell death, such as apoptosis, necroptosis and DNA damage related to cell injury $(23,24)$. Thus, we executed models of cell death induced by various cell death inducers: methyl methanesulfonate (MMS, a DNA-damaging reagent), $\mathrm{H}_{2} \mathrm{O}_{2}$ (oxidative damage), TNF- $\alpha$ plus cycloheximide (apoptotic challenge) and TNF- $\alpha$ plus z-VAD-fmk (necroptosis stimulation) using YAMC cells. Briefly, cells were subjected to transfection with siSirt6/siControl, pSirt6/pVector or siRspo1/siControl using the protocol described above. After $24 \mathrm{~h}$, the transfected cells were reseeded in 96-well, white-walled, clear-bottomed plates at $1.5 \times 10^{4}$ density per well and cultured with IFN- $\gamma$-free medium at $37^{\circ} \mathrm{C}$ for $24 \mathrm{~h}$, followed by treatment with adequate cell-death-inducer cocktails: MMS (2 mM), $\mathrm{H}_{2} \mathrm{O}_{2}(400 \mu \mathrm{M}), \mathrm{TNF}-\alpha$ (20 ng/mL) + cycloheximide $(5 \mu \mathrm{g} / \mathrm{mL})$ and TNF- $\alpha(20 \mathrm{ng} / \mathrm{mL})+\mathrm{z}$-VAD-fmk $(20 \mu \mathrm{M})$. All cell-death-inducer cocktails were prepared in IFN- $\gamma$-free medium. For MMS treatment, cells were challenged with MMS for $1 \mathrm{~h}$, followed by 
culture with MMS-free medium for an additional $24 \mathrm{~h}$. For other treatment regimens, cells were treated with adequate cell-death-inducer cocktails for $24 \mathrm{~h}$. At the end of treatments, cell viability was assessed using CellTiter-Glo Assay Kit (Promega, Madison, WI, USA), following the manufacturer's protocol.

\section{RNA Sequencing}

Total RNA was extracted from YAMC cells using RNeasy Micro Kit (Qiagen, Hilden, Germany) according to the manufacturer's instructions. RNA integrity and quantity were assessed using Agilent 2100 Bioanalyzer (Agilent Technologies, Santa Clara, CA, USA). PolyA-enriched RNA was purified from total RNA using paramagnetic oligo-dT beads, fragmented and used for constructing cDNA libraries with NEBNext Ultra RNA Library Prep Kit for Illumina following the manufacturer's protocol (NEB, Ipswich, MA, USA). Libraries were amplified, followed by removing primers prior to sequencing. These libraries were sequenced on Illumina HiSeq 2500 generating 100 bp paired end reads (Peking Jabrehoo Med Tech Co., Beijing, China). After sequencing, raw reads were cleaned by removing adaptor sequences, empty reads and low-quality sequences. The reads that passed filtering were aligned to reference sequences of the mouse transcriptome (Ensembl release 75) using the Bowtie version 1.0.1 alignment tool. Read counts were normalized using edgeR, and reads per kilobase per million mapped reads values were calculated as the number of counted reads per 1000 mapped and counted bases per geometric mean of normalized read counts per million. Fold change ratio between the two groups was calculated by reads per kilobase per million mapped reads, and an absolute value of $\log 2$ ratio $\geq 0.415$ was used as a threshold to judge the difference of gene expression. Heatmaps of differentially expressed genes were generated by the GENE-E visualization and analysis platform. Gene ontology term analysis was performed using Gene Set Enrichment
Analysis software $(25,26)$ and visualized by the REViGO tool (27).

\section{Measurement of Myeloperoxidase Activity \\ Briefly, colon tissues were homoge-} nized in $250 \mu \mathrm{L}$ myeloperoxidase (MPO) assay buffer and centrifuged at $13000 \mathrm{~g}$ for $10 \mathrm{~min}$ at $4^{\circ} \mathrm{C}$ to remove insoluble material. The supernatants were processed for measurement of MPO activity using an MPO Colorimetric Activity Assay Kit (Sigma-Aldrich), following the manufacturer's protocol. MPO activity was normalized by the protein loading in each sample and reported as unit/gm protein.

\section{Cytokine and Chemokine Measurements}

Plasma cytokine/chemokine measurements were made using Meso Scale Diagnostics (MSD, Gaithersburg, MD, USA) multiplex array technology. Briefly, cytokines and chemokines were measured using a mouse-specific 96well multispot plate of V-PLEX Plus Proinflammatory Panel 1 Kit (MSD), which included five markers: IL-1 $\beta$, IL-6, IL-10, TNF- $\alpha$ and KC. The assay was performed according to the manufacturer's instructions. A total volume of $50 \mu \mathrm{L}$ diluted (1:2.5) sample was used in each well. The assay was run in duplicate for each sample. Plate readings were performed on a SECTOR Imager 2400 instrument (MSD). Raw data were analyzed using MSD Discovery Workbench software (version 4.0). The curves were fitted using a $4 \mathrm{PL}$ fit with $1 / \mathrm{y}^{2}$ weighting according to the manufacturer's instructions, and concentrations were determined from the standard curves.

\section{Statistical Analysis}

All experiments were performed at least twice. Statistical analysis was performed with Graphpad Prism 6 software. Reported data are the mean \pm standard error of the mean, and statistical significance was assessed with either the Student $t$ test or one-way analysis of variance followed by Fisher's least significant difference post hoc test. $P<0.05$ was considered significant.

\section{Web Deposition of Data}

Data in this study have been deposited in the Gene Expression Omnibus site (www.ncbi.nlm.nih.gov/geo), accession number GSE89620.

\section{RESULTS}

\section{Sirt6 Is Highly Expressed in Mouse Intestines and Predominantly Localized to Intestinal Epithelial Cells in the Crypt Compartment}

We first thought to compare expression patterns of Sirt6 protein in mouse tissues. Using Western blot, we found that Sirt6 protein was widely expressed in different tissues, with particularly strong expressions in the thymus, spleen and intestines (Figure 1A). To further characterize Sirt6 expression in intestines, we examined its cellular localization in the mouse intestines using immunofluorescent staining. While it was strongly detected in crypt epithelial cells (Figure 1B), Sirt6 was weakly presented in other intestinal epithelial cells, suggesting its role in the regulation of intestinal epithelial cell homeostasis.

\section{Colitis Is Associated with Downregulation of Colonic Sirt6 Expression in Mice and Humans}

To examine whether acute colitis affected Sirt6 expression in mouse colons, adult mice (male, 8-10 wks old) were given $3.5 \%$ DSS in drinking water ad libitum for a period of $7 \mathrm{~d}$ to achieve acute experimental colitis using our standard protocol (14). As expected, DSS administration induced experimental colitis in the mice (Supplementary Figure S1). Development of DSS-induced colitis was associated with downregulation of Sirt6 protein (Figure 2A) in a timedependent manner in mice. Notably, Western blot revealed that some lowmolecular-weight bands were presented in colonic tissue samples derived from DSS-treated mice, suggesting that DSSinduced colitis may result in degradation 
A
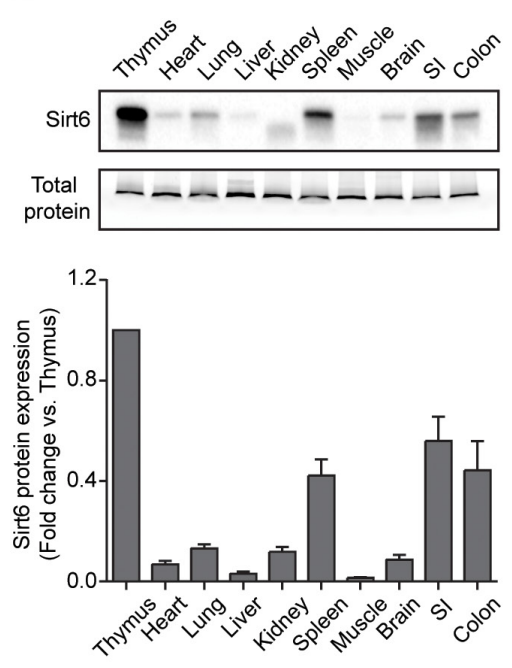

B
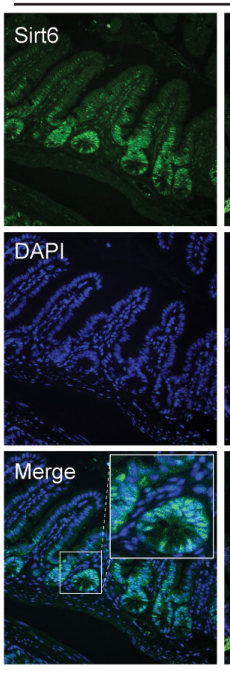

Small intestine
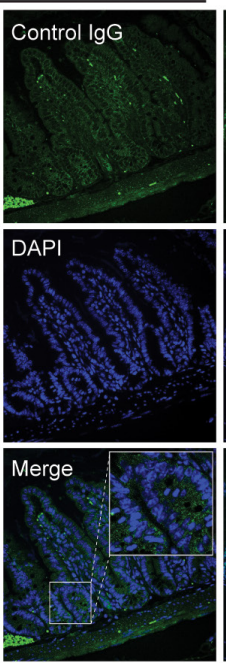

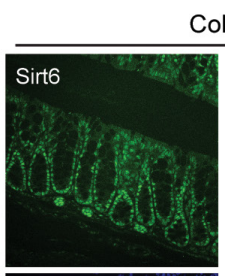

Colon

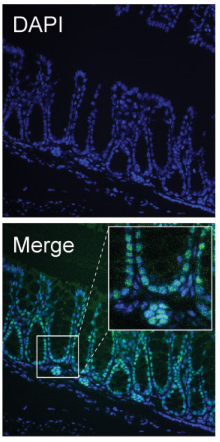

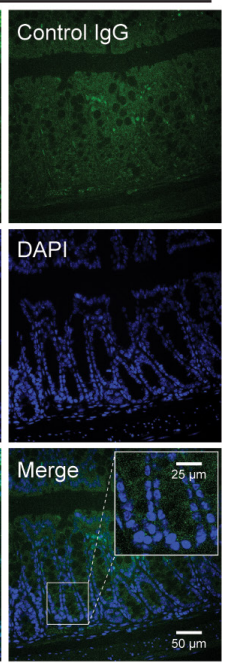

Figure 1. Characterization of Sirt6 protein expression in mouse tissues. (A) Western blot analysis of Sirt6 protein expression in various mouse tissues. Total proteins isolated from indicated mouse tissues ( $10 \mu \mathrm{g} / \mathrm{lane}$ ) were subjected to electrophoresis and Western blot using rabbit antibody against mouse Sirt6, as described in Methods. Upper panel: autoradiograph of a representative immunoblot and total protein gel. Lower panel: densitometric analysis of the immunoblot data (normalized to total protein, $n=3$ ). Results are expressed as mean \pm standard error of the mean (SEM). (B) Cellular localization of Sirt6 in mouse intestines. Deparaffinized sections of the small and large intestines of normal mice were stained with immunofluorescence using rabbit antibody against murine Sirt6 or rabbit naïve lgG, as indicated. Nuclei were counterstained with DAPI. Sections were observed by immunofluorescence microscopy. Sl, small intestine.

A

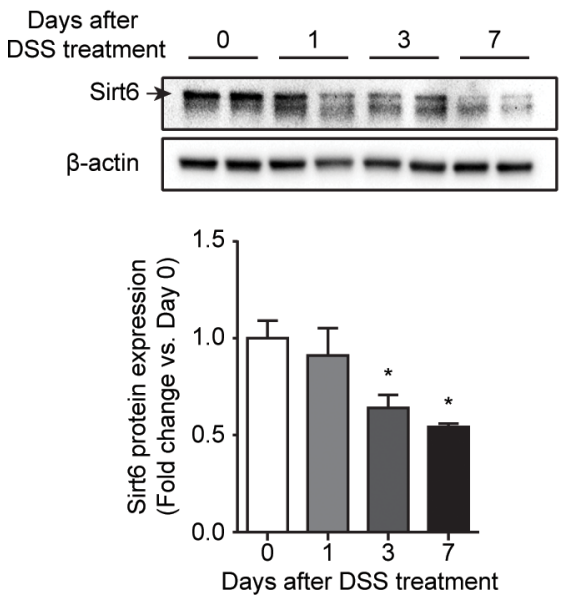

Figure 2. Colitis is associated with downregulation of colonic Sirt6 expression in mice and humans. For (A) the mouse colitis model, wild-type C57BL/6J mice (male, 8-10 wks old) were given drinking water containing $3.5 \%$ DSS for up to $7 \mathrm{~d}$. Colonic tissues were collected at the indicated time points ( $n=5$ per time point). For $(B)$ human samples, mucosal biopsies from the sigmoid colons of health individuals $(n=17)$ and UC patients $(n=15)$ were obtained through diagnostic colonoscopy procedures. Tissue samples were processed for assessment of expression of Sirt6 protein with (A) Western blot or (B) mRNA, as described in Materials and Methods. (A) Sirt6 protein levels in colon during development of DSS-induced colitis in mice. Upper panel: autoradiograph of a representative immunoblot. Lower panel: densitometric analysis of the immunoblot data (normalized to $\beta$-actin). (B) Relative expression of SIRT6 mRNA in the sigmoid colon biopsies of healthy individuals and UC patients. Results are expressed as mean \pm SEM. ${ }^{*}, P<0.05$ versus $0 \mathrm{~d}$. of intestinal Sirt6 protein. Furthermore, we examined intestinal SIRT6 expression in patients with UC and healthy individuals. UC patients $(41.4 \pm 13.6$ years old, 7 male and 8 female) with Mayo Endoscopic Score between 1 and 3 were recruited for this study. Among the healthy individuals, 7 were male and 10 were female, $47.6 \pm 12.0$ years old. Interestingly, we found that the mean value of SIRT6 mRNA in inflamed mucosa of UC patients was significantly lower than that in colon tissues of healthy individuals (Figure 2B), suggesting a decrease in colonic SIRT6 gene expression at the transcriptional level in UC patients.

\section{IFN- $\gamma$ Attenuates Sirt6 Protein Expression in Colonic Epithelial Cells}

In this experiment, we examined whether inflammatory mediators modulated Sirt6 expression in colonic epithelial cells. Evidence shows that IFN- $\gamma$ is a dominant proinflammatory cytokine in colitis $(28,29)$. Thus, we first studied the effect of IFN- $\gamma$ treatment on Sirt6 expression in YAMC cells, an epithelial cell line derived 
from mouse colonic epithelial cells. We found that Sirt6 protein expression in YAMC cells was markedly inhibited by IFN- $\gamma$ treatment (Figure 3A). As free-radical species are generated during inflammation and affect epithelial cell survival, we determined the effect of hydrogen peroxide $\left(\mathrm{H}_{2} \mathrm{O}_{2}\right.$, oxidative free radicals) challenge on Sirt6 expression in YAMC cells. Similar to IFN- $\gamma$, treatment with $\mathrm{H}_{2} \mathrm{O}_{2}$ downregulated Sirt6 protein expression in YAMC cells in a dose-dependent manner (Figure 3B). Furthermore, we confirmed that IFN- $\gamma$ inhibited SIRT6 expression in human intestinal epithelialspecific cells (IECs) using HT-29, a human colonic epithelial cell line (Figure 3C).

\section{Sirt6 Plays a Role in Maintaining the Resistance of Intestinal Epithelial Cells to Cell Death Challenges}

Previous studies showed that Sirt6 is a master epigenetic gatekeeper of glucose metabolism, a fundamental cellular event generating metabolic intermediates important for cell growth (30). However, little is known about the role of Sirt6 on intestinal epithelial cell homeostasis. Thus, using both loss-of-function and gain-of-function approaches, we investigated whether Sirt6 regulates intestinal epithelial cell resistance to cell death.
We found that when Sirt6 expression was silenced by transfection with siSirt6, a siRNA against Sirt6 transcripts (Figure 4A), YAMC cells had increased cell death in response to various inducers, including MMS (a DNA-damaging reagent), $\mathrm{H}_{2} \mathrm{O}_{2}$ (oxidative damage), TNF- $\alpha$ plus cycloheximide (apoptotic challenge) and TNF- $\alpha$ plus z-VAD-fmk (necroptosis stimulation) (Figure 4B).

In addition, when Sirt6 was overexpressed by transfection with $p I R E S 2-$ zsGreen1-mSirt6, an expression plasmid construct containing the full open reading frame of mouse Sirt6 cDNA (Figure 4C), YAMC cells were markedly resistant to cell death induced by DNA damage, superoxides, apoptotic stimulation and necroptosis signals (Figure 4D). Taken together, our data suggest that Sirt6 renders intestinal epithelial cells resistant to cell death insults.

\section{Intestinal Epithelial Cell-specific Knockout of Sirt6 Increases Susceptibility to DSS-induced Colitis in Mice}

In this study, we first investigated the physiological impact of downregulation of Sirt6 expression in the intestinal epithelium of mice using a tissue-specific knockout approach. Sirt6 $6^{\mathrm{Co} / \mathrm{Co}}$ mice
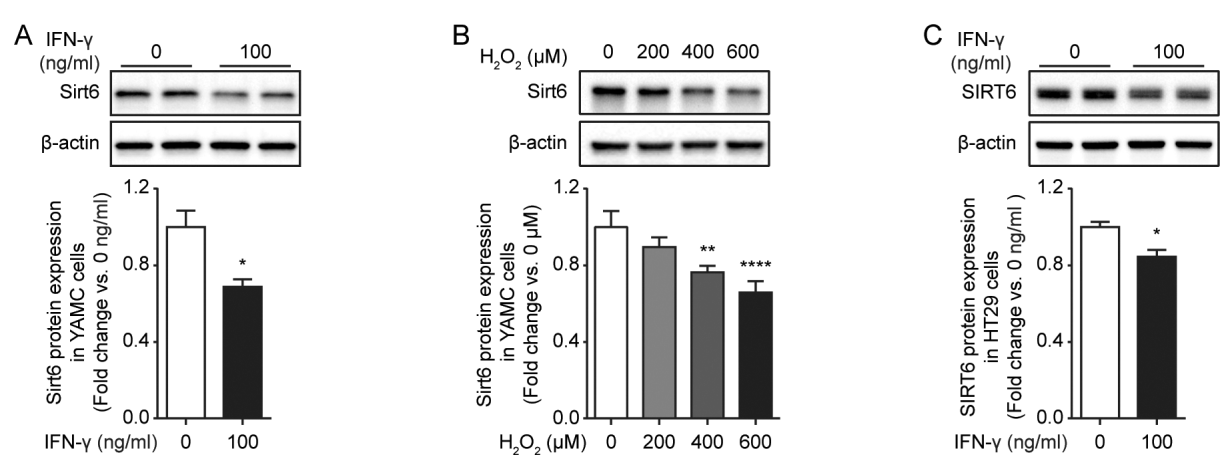

Figure 3. Inflammatory mediators attenuate Sirt6 protein expression in mouse and human colonic epithelial cells. (A, B) YAMC and (C) HT-29 cells were subjected to treatments as indicated. After 24 and $48 \mathrm{~h}$, respectively, total cellular proteins were extracted from harvested cells. Levels of Sirt6 protein were accessed with Western blot using anti-Sirt6 antibody, as described in Materials and Methods. Upper panels illustrate the autoradiographs of representative immunoblots; lower panels show densitometric analysis of the immunoblot data (normalized to $\beta$-actin). The experiments were performed three times. Results are expressed as mean \pm SEM. $n=3$ in each group. ${ }^{*}, P<0.05$ versus control; ${ }^{* *}, P<0.01$ versus control; ${ }^{* * *}, P<0.0001$ versus control.

(a mouse line harboring a loxP-flanked Sirt6 allele [13]) were crossed with

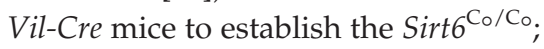
Vil-Cre $^{+/}$(ie, Sirt6 ${ }^{I E C-K O}$ ) mouse line. Genotyping analysis of tail biopsy samples revealed that Sirt6 ${ }^{I E C-K O}$ mice were characterized as heterozygous for $\mathrm{Vil}-\mathrm{Cre}$ transgene and homozygous for floxed Sirt6 allele (Supplementary Figure S2A). Using Western blot, we found that Sirt6 ${ }^{\text {IEC-KO }}$ mice lacked Sirt6 protein expression in an intestinal tissue-specific manner (Supplementary Figure S2B). The Sirt6 ${ }^{I E C-K O}$ mice were fertile and healthy. Their body weights were indistinguishable from those of their control littermates (Supplementary Figure S2C). Histological examination showed that Sirt $6^{I E C-K O}$ mice did not display any phenotypical abnormalities in their intestines (Supplementary Figure S2D). Together, the data suggest that Sirt6 expression is not essential for orchestrating morphogenesis and maintaining integrity in intestinal epithelial cells under a normal physiological state.

Next, we examined whether lack of Sirt6 in the intestinal epithelium affected colitis development. For this purpose, Sirt ${ }^{I E C-K O}$ mice and their control littermates were given 3.5\% DSS in drinking water ad libitum for $7 \mathrm{~d}$ to induce colitis. We found that DSS treatment resulted in greater weight loss (Figure 5A) and more severe colitis (Figure 5B) in $\operatorname{Sirt6}^{I E C-K O}$ mice than that in their control littermates ( $\mathrm{n}=14-17$ mice per group, $P<0.05$ ), specifically in the early period of colitis development. Histological examination showed that Sirt6 ${ }^{I E C-K O}$ mice displayed more profound signs of colitis at $\mathrm{d} 7$ than their control littermates (Figure 5C). Microscopic scores for DSS-induced colonic inflammation (Figure 5D) and cryptepithelial injury (Figure 5E) were significantly greater in Sirt6 ${ }^{I E C-K O}$ mice than that in their control littermates. Furthermore, we found that there was significantly higher MPO activity in the colons of Sirt $6^{I E C-K O}$ mice with DSS-induced colitis compared with their control littermates (Figure 5F). Together, our data suggest that the loss of Sirt6 in intestinal 
A

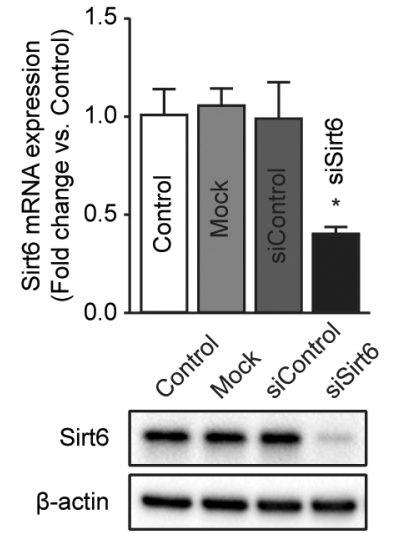

C

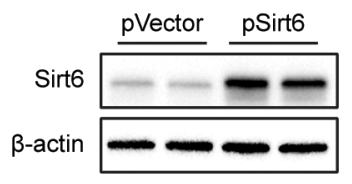

B
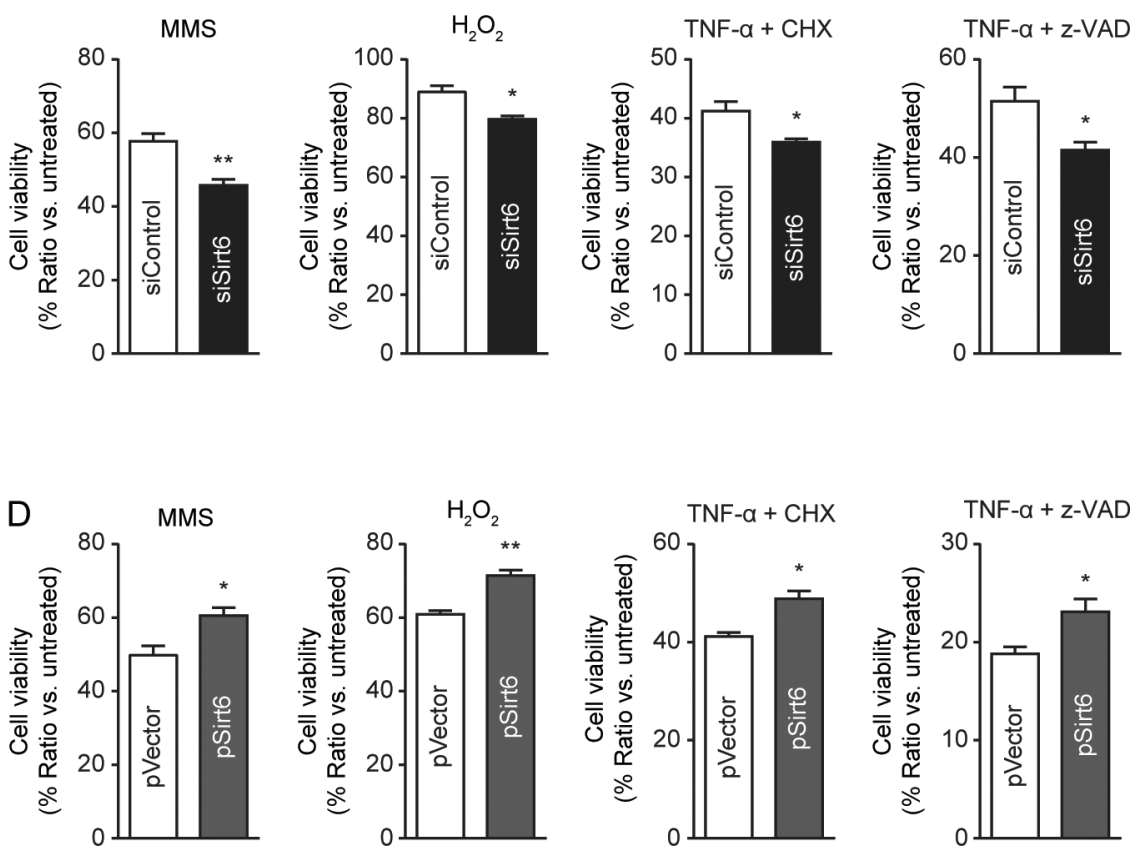

Figure 4. Downregulation of Sirt6 results in increased susceptibility of mouse colonic epithelial cells to cell death challenges, whereas Sirt6 overexpression increases resistance of mouse colonic epithelial cells to cell death challenges. (A) Silencing of Sirt6 expression in colonic epithelial cells was achieved using siRNA technology. The Sirt6 knockdown in vitro was confirmed using qRT-PCR (upper panel) and Western blot (lower panel). (B) Silencing of Sirt6 increases susceptibility of colonic epithelial cells to cell death challenges. YAMC cells were subjected to transfection with siSirt6 or siControl, as described above. Forty-eight hours later, cells were treated with indicated treatments. Twenty-four hours after being challenged with cell death inducers, cell viability was assessed using CellTiter-Glo assay. The experiments were performed three times. Results are expressed as mean \pm SEM. $n=3$ in each group. ${ }^{*}, P<0.05$ versus control; ${ }^{* *}, P<0.01$ versus control. (C) Ectopic expression of Sirt6 in intestinal epithelial cells by transfection with pSirt6 (ie, plRES2-zsGreen 1-mSirt6 plasmid). Sirt6 overexpression was confirmed with Western blot. pVector, the plRES2-zsGreen 1 plasmid (ie, control). (D) Overexpression of Sirt6 renders colonic epithelial cells resistant to cell death challenges. YAMC cells were subjected to transfection with pSirt6 or pVector, as described in panel C. Forty-eight hours later, they were subjected to indicated treatments. Twenty-four hours later, cell viability was assessed using CellTiter-Glo assay. Results are expressed as mean \pm SEM and represent average of findings from three independent experiments. $\mathrm{n}=3$ in each group. ${ }^{*}, P<0.05$ versus control; ${ }^{* *}, P<0.01$ versus control.

epithelial cells markedly exacerbates

DSS-induced colitis.

\section{Sirt6 Plays an Important Role in Sustaining R-spondin-1 Expression in Intestinal Epithelial Cells under Inflammation}

It has been reported that Sirt6 modulates cellular homeostasis via shaping of diverse transcriptional networks $(31,32)$. To determine whether Sirt6 is capable of influencing gene expression in intestinal epithelial cells under physiological and inflammatory conditions, we knocked down Sirt6 using siRNA-mediated gene silencing in YAMC cells. Then, we performed RNA-seq transcriptome analysis in naïve and TNF- $\alpha$-treated Sirt6-silenced YAMC cells to enrich for direct Sirt6 targets associated with inflammatory stimulation. By comparing Sirt6-silenced YAMC cells to siControl-transfected YAMC cells, we found that there were 916 (Supplementary Tables S1 and S3) and 1026 (Supplementary Tables S2 and S3) differentially expressed genes in the naïve and TNF- $\alpha$ treatment conditions, respectively (Figure 6A). We also found that there were 302 genes that were co-expressed differentially in both naïve and TNF- $\alpha$ treatment conditions between Sirt6-silenced and siControl-transfected YAMC cells (Figure 6A and Supplementary Table S3).
Among the differentially expressed genes, we found numerous targets related to cell proliferation in cells treated with TNF- $\alpha$ (Figure 6B). The hierarchical clustering revealed that Sirt6 silencing markedly affected global gene transcription in intestinal epithelial cells under naïve and TNF- $\alpha$ treatment conditions (Figure 6C). Notably, the differential gene expression analysis indicates downregulation of $R$-spondin-1 (Rspo1) in TNF- $\alpha$-treated Sirt6-knockdown YAMC cells (Figure 6C).

Rspo1 is a Wnt signaling pathwayassociated mitogen $(33,34)$. Previously, Zhao et al. showed that Rspo1 ameliorates DSS-induced colitis in mice (35). Thus, we further verified the effect of 

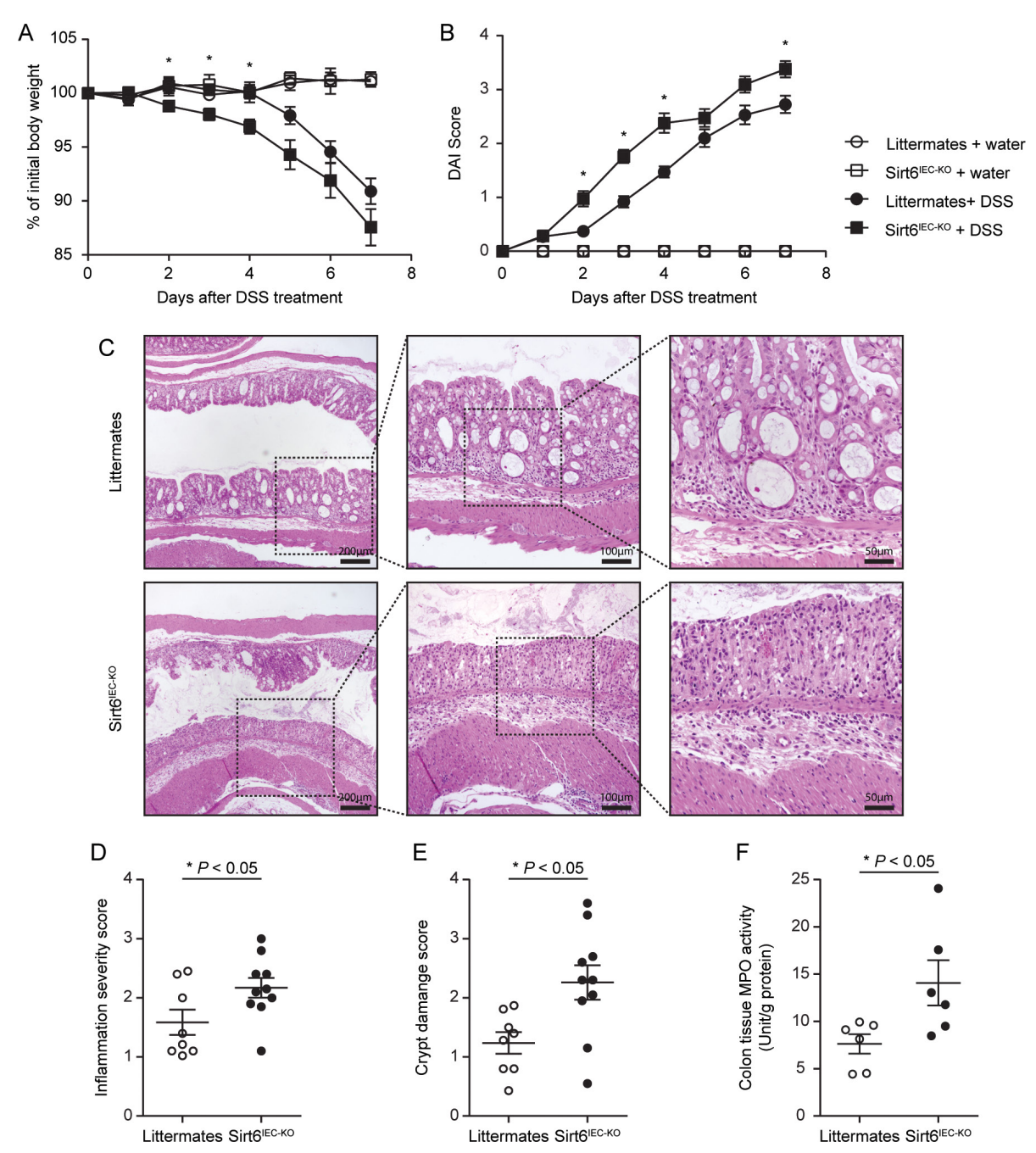

Figure 5. Intestinal epithelial cell-specific knockout of Sirt6 gene increases susceptibility to DSS-induced colitis in mice. Sirt6 ${ }^{I E C-K O}$ mice $(n=14)$ and their control littermates $(n=17)$ (male, $8-10$ wks old) were given drinking water containing 3.5\% DSS for $7 \mathrm{~d}$. A set of mice received normal drinking water as controls. (A) Body weight and (B) disease activity index (DAI) were monitored on a daily basis. Mice were euthanized at $d 7$ of DSS treatment. (C) Colonic tissues were processed for routine histology and H\&E staining and assessed in a double-blind fashion for histological colonic tissue injury in terms of (D) inflammation severity and (E) crypt of damage using a scoring system described in Materials and Methods. (F) The severity of colitis was further determined by MPO assay. Results are expressed as mean \pm SEM. ${ }^{*}, P<0.05$ versus control.

TNF- $\alpha$ on Rspo1 expression in YAMC cells using qRT-PCR and Western blot. We confirmed that reduction of Rspo1 mRNA and protein levels occurred in Sirt6-silenced IECs after TNF- $\alpha$ treatment in vitro (Figures $7 \mathrm{~A}$ and $7 \mathrm{~B}$ ). In addition, knocking out Sirt6 in intestinal epithelial cells in vivo did not affect intestinal Rspo1 expression at baseline (Figure 7C). In contrast, treatment of Sirt6 $6^{I E C-K O}$ mice with lipopolysaccharide resulted in downregulation of Rspo1 mRNA (Supplementary Figure 3) and protein expression in colons (Figure 7C). Similarly, we found a trend of decreasing Rspo1 protein levels in colons of wildtype littermate mice with DSS-induced colitis (Figure 7D). Furthermore, a significant reduction of Rspo1 protein expression was observed in Sirt6 ${ }^{I E C-K O}$ mice compared with control littermates under a colitis condition (Figure 7D). Finally, we performed an in vitro study to examine whether Rspo1 mediates the protective role of Sirt6 that was revealed in the experiments described in Figure 4. We found that silencing Rspo1 with siRNA technology resulted in diminishing the beneficial effect of Sirt6 overexpression on protection of YAMC cells against cell death induced by MMS, $\mathrm{H}_{2} \mathrm{O}_{2}$, TNF- $\alpha$ plus cycloheximide and TNF- $\alpha$ plus z-VAD-fmk (Figure 8). Collectively, our data suggest that Sirt6 has an important role in protecting intestinal epithelial cells under inflammation by maintaining expression of the Rspo1 gene.

\section{DISCUSSION}

In the present study, we investigated the role of intestinal epithelial Sirt6 in gut injury and inflammation. Our findings show that Sirt6 is expressed by intestinal epithelial cells, predominantly in crypt cells. We demonstrated that colitis is associated with decreased levels of intestinal Sirt6. Inflammatory mediators, including IFN- $\gamma$ and reactive oxygen species, directly inhibited Sirt6 expression in intestinal epithelial cells. Mice deficient in Sirt6 in intestinal epithelial cells are prone to DSS-induced colonic mucosal injury, suggesting that inflammationinduced impairment of Sirt6 expression in intestines contributes to the pathogenesis of colitis. Furthermore, we found for the first time that Sirt6 is critical in maintaining levels of Rspo1 in intestinal epithelial cells under inflammatory conditions. Since Rspo1 is an important trophic factor for intestinal epithelial cell growth $(35,36)$, our data strongly suggest the notion that Sirt6 plays a central role in protecting against colitis-associated intestinal epithelial cell injury via preservation of Rspo1.

Emerging studies have shown that Sirt6 is a key regulator of genome stability, glucose homeostasis, metabolism and inflammation (9). It governs diverse cellular functions, such as transcription, telomere integrity and DNA repair (9). Mouse pups with whole-body Sirt6 
A

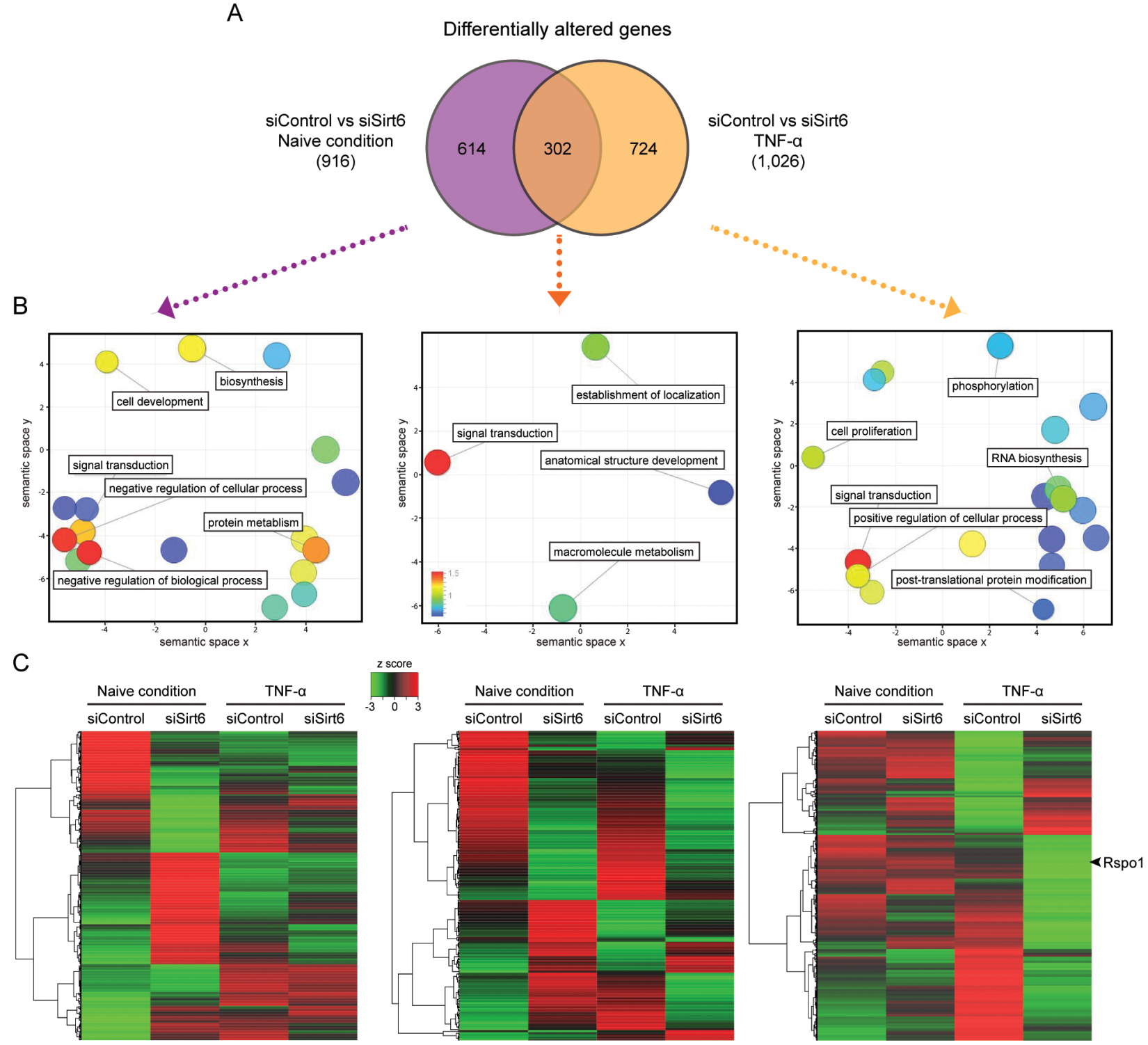

Figure 6. Abolishing Sirt6 expression impacts transcriptome responses to TNF- $\alpha$ stimulation in colonic epithelial cells. YAMC cells were transfected with Sirtb siRNA as described in Materials and Methods. Forty-eight hours after transfection, cells were cultured with medium containing TNF- $\alpha(100 \mathrm{ng} / \mathrm{mL})$ or medium alone for an additional $24 \mathrm{~h}$. At the end of treatment, cells were harvested for RNA extraction, followed by RNA-seq transcriptome analysis. (A) Differential gene expression analysis (log2 ratio $\geq 0.415$ fold change) was carried out to determine genes that were altered in YAMC cells transfected with sisirt6 compared with scramble siRNA (siControl) under either nailve condition (purple circle) or TNF- $\alpha$ stimulation (orange circle). Venn diagram demonstrates the number of shared or specific genes of each group of cells. (B) Analysis of Gene Ontology (GO) terms to identify enriched GO terms associated with Sirtb silencing. The genes from each category in the Venn diagram were subjected to $G O$ analysis to identify GO terms enrichment using GESA software. Results are illustrated as a scatterplot using the REViGO visualization tool. The circle size is proportional to the frequency of the GO term, while the color represents the normalized enrichment score generated from GESA (red: higher; blue: lower). (C) The genes from each category in the Venn diagram were further subjected to hierarchical cluster analysis of common differentially expressed genes associated with Sirto silencing in nailve and TNF- $\alpha$ stimulation conditions.

deficiency exhibit postnatal growth retardation and eventually die at about 4 wks after birth, highlighting the importance of Sirt6 for growth and survival (11).
Furthermore, a distinctive phenotype of colitis with prominent epithelial sloughing was previously found in weaning Sirt6-null mice (11), suggesting that Sirt6 is required in remodeling tissue architecture as intestinal mucosa grow and mature during postnatal development. However, we did not find a significant 


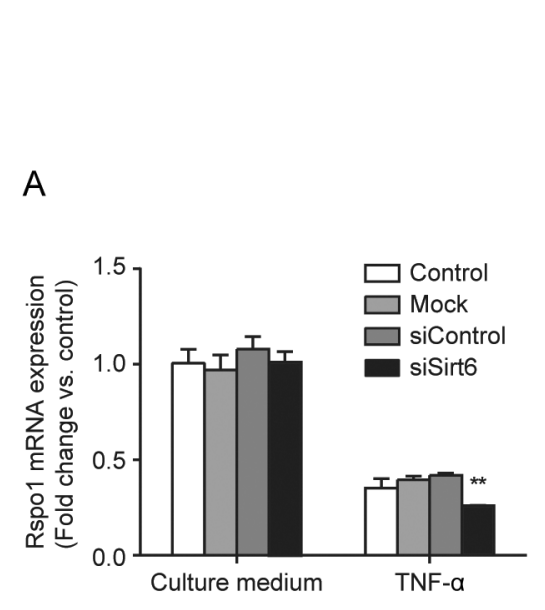

B
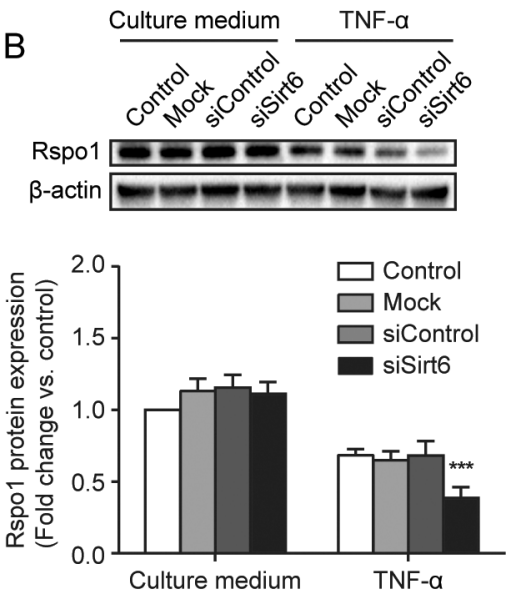

C
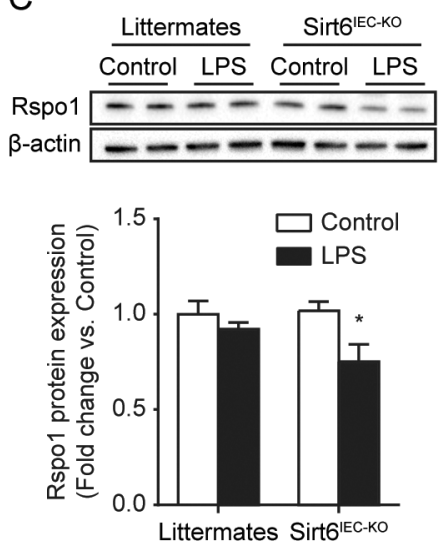
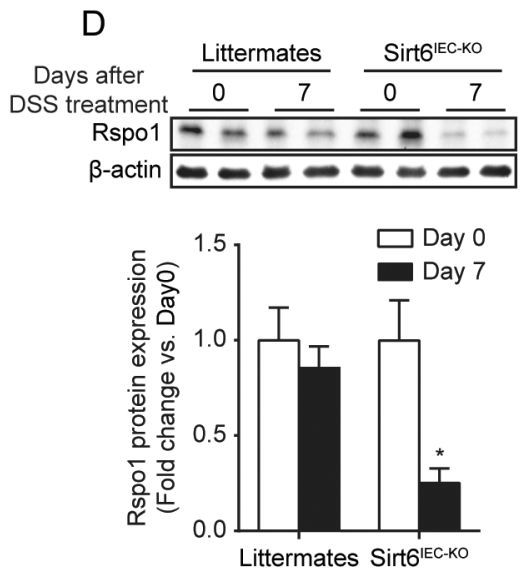

Figure 7. Sirt6 is required to maintain Rspol expression of intestinal epithelial cells under inflammation status. ( $A$ and B) YAMC cells ( $n=3$ in each group) were transfected with Sirt6 siRNA (siSirt6, $50 \mathrm{nM}$ ) or negative control siRNA (siControl, $50 \mathrm{nM}$ ) using lipofectamine 2000. Forty-eight hours after transfection, cells were cultured with medium containing TNF- $\alpha(100 \mathrm{ng} / \mathrm{mL})$ or medium alone for an additional $24 \mathrm{~h}$. At the end of treatment, cells were harvested to measure expression of (A) Rspo 1 mRNA with quantitative real-time RT-PCR and (B) Rspol protein with Western blot. (C) Sirt6 IEC-KO mice and their control littermates (male, 8-10 wks old, $n=4$ in each group) were intraperitoneally injected with either LPS ( $2 \mathrm{mg} / \mathrm{kg}$ ) or saline ( $100 \mu \mathrm{L}$, sham control). Twenty-four hours after treatment, mice were euthanized by $\mathrm{CO}_{2}$ inhalation. Then, colons were collected and processed to determine levels of Rspo 1 protein by Western blot. (D) Western blot examination of Rspo 1 protein expression in Sirt6 ${ }^{I E C-K O}$ mice and their control littermates (male, 8-10 wks old, $n=4$ in each group) with DSS-induced colitis. Colonic tissues were collected on d 7 after induction of colitis with 3.5\% DSS drinking water. Results are expressed as mean \pm SEM and represent the average of findings from three independent experiments. ${ }^{*}, P<0.05$ versus control; ${ }^{* *}, P<0.01$ versus control; ${ }^{* *}, P<0.001$ versus control.

effect of intestinal epithelial deletion of Sirt6 on intestinal morphology in mice, implying that Sirt6 in intestinal epithelial cells is not an essential protein for intestinal development and homeostasis in normal physiological states.
Evidence suggests that Sirt6 expression is inhibited in numerous pathological conditions associated with inflammation. For example, decreased Sirt6 expression in fetal membranes occurs in preterm labor (37). Balestrieri et al. found that

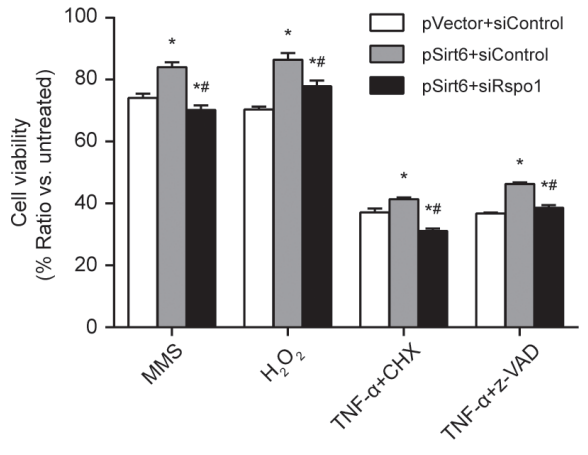

Figure 8. Rspol is required for protection of intestinal epithelial cells against cell death challenges by Sirt6 overexpression. YAMC cells were subjected to treatment as indicated using a protocol described in Figure 4. At the end of treatment, cells were processed to measure cell viability using CellTiter-Glo assay. Results are expressed as mean \pm SEM. $n=4$ in each group. ${ }^{*}, P<0.05$ versus pVector + siControl; \#, $P<0.05$ versus pSirt6 + siControl.

Sirt6 protein expression is downregulated in diabetic atherosclerotic lesions (38). A previous study identified that $\mathrm{H}_{2} \mathrm{O}_{2}$ treatment results in marked reduction of Sirt6 in endothelial cells (39). Consistent with these previous findings, our data indicate that mucosal Sirt6 protein expression is decreased in colitis. Furthermore, we found that not only $\mathrm{H}_{2} \mathrm{O}_{2}$, but also IFN- $\gamma$ is a strong inhibitor of Sirt6 expression in intestinal epithelial cells. In addition, our study shows that deletion of Sirt6 in intestinal epithelial cells exacerbates DSS-induced colitis, strongly supporting the notion that inflammation-induced downregulation of intestinal epithelial Sirt6 directly impacts intestinal mucosal integrity during colitis development.

Sirt6 is an enzyme that has ADPribosyltransferase activity $(40,41)$ and histone deacetylase activity (42-45). It influences chromatin structure and gene expression. Particularly, previous studies demonstrated that Sirt6 functions at chromatin to attenuate NF- $\kappa \mathrm{B}$-dependent transcription (46). On the other hand, Sirt6 was found to positively regulate TNF- $\alpha$ production at the post-transcriptional level in vitro (47). Thus, the effect 
of downregulation of Sirt6 in regulating the inflammatory response may be complicated in vivo. Indeed, our data show that knockout of Sirt6 in intestinal epithelial cells did not influence the cytokine profile in colitis (Supplementary Figure S4), while the Sirt6 ${ }^{I E C-K O}$ mice were more susceptible to DSS-induced colitis than their control littermates. This observation suggests that Sirt6 protects intestinal epithelial cells against inflammatory injury through a cytokine-independent mechanism. Using global RNA expression data obtained by RNA-seq transcriptome analysis, we found that knockdown of Sirt6 markedly alters gene expression in naïve intestinal epithelial cells. Furthermore, we found that the response to TNF- $\alpha$ challenge in Sirt6-silenced IECs differs from that in IECs with intact Sirt6 expression. Together, it appears that Sirt6 plays an important role in maintaining the homeostasis of gene expression in intestinal epithelial cells in both the normal physiological state and inflammation.

In addition, our finding that knocking down Sirt6 expression in intestinal epithelial cells decreases Rspo1 expression in inflammation is intriguing. Rspo1 is a trophic factor for intestinal epithelial cells $(35,36)$. Previously, Zhao et al. (35) reported that Rspo1 is predominantly expressed in crypt Paneth cells and differentiated enterocytes along the intestinal villus epithelium, where Sirt6 is also expressed in the mouse gut. Wnt/ $\beta$ catenin signaling plays an essential role in regulating gastrointestinal homeostasis. Rspo1 has been shown to stimulate intestinal epithelial cell proliferation and protect epithelial cells from constant exposure to toxins, inflammatory cytokines and reactive oxygen radicals by increasing $\beta$-catenin nuclear localization and subsequently turning on Wnt downstream target gene activation $(35,48,49)$. Administration of recombinant Rspo1 was reported to strongly ameliorate colitis symptoms in several experimental colitis mouse models $(35,48,50)$, suggesting that it has a protective role in the gut under inflammation. We show here that a decrease in Rspo1 expression is associated with an increase in sensitivity to various death inducers in Sirt6 knockdown cells. Sirt6 ${ }^{I E C-K O}$ mice exhibit a lack of ability to maintain Rspo1 expression in their intestines under inflammation, implying that an increase in susceptibility to inflammatory intestinal injury in Sirt6 ${ }^{I E C-K O}$ mice may be due to a lack of the protective factor Rspo1. Furthermore, we found that Rspo1 mediates the role of Sirt6 in the protection of intestinal epithelial cells against cell injury. Together, our data in combination with previous findings support the notion that the Sirt6-Rspo1 signal axis plays a role in the protection of intestinal epithelial cells against inflammatory injury.

\section{CONCLUSION}

In summary, our study reveals that expression of Sirt6 in intestinal epithelial cells is necessary for protection of the intestinal mucosa against colitis. We identified that intestinal inflammation causes downregulation of Sirt6 in the gut, which leads to increased susceptibility of intestinal epithelial cells to injury. In addition, we found Rspo1 to be regulated by Sirt6 in intestinal epithelial cells. Our data suggest that inflammation-induced downregulation of Sirt6 contributes to decreased Rspo1 expression in intestinal epithelial cells, thus leading to an increased susceptibility of the intestinal mucosa to injury under inflammatory conditions. These findings provide novel insights into both the pathogenesis of colitis and potential therapeutic targets for intestinal injury and inflammation.

\section{DISCLOSURE}

The authors declare that they have no competing interests as defined by Molecular Medicine or other interests that might be perceived to influence the results and discussion reported in this paper.

\section{ACKNOWLEDGMENTS}

This study was supported by National Institute of Diabetes and Digestive and Kidney Diseases grant R01DK064240 (XDT), National Institute of General Medical Sciences grant R01GM117628
(XDT), Eunice Kennedy Shriver National Institute of Child Health and Human Development grant R01HD060876 (IDP), US Department of Veterans Affairs Merit Award I01BX001690 (XDT), the Dorothy $M$ and Edward E Burwell Professorship (XDT) and Ministry of Health of the People's Republic of China Special Project grant 201002020 (JQ). The sponsors had no role in study design or collection, analysis or interpretation of the data.

\section{REFERENCES}

1. Cosnes J, Gower-Rousseau C, Seksik P, Cortot A. (2011) Epidemiology and natural history of inflammatory bowel diseases. Gastroenterology. 140:1785-94.

2. Kaplan GG. (2015) The global burden of IBD: from 2015 to 2025. Nat. Rev. Gastroenterol. Hepatol. 12:720-27.

3. Molodecky NA, et al. (2012) Increasing incidence and prevalence of the inflammatory bowel diseases with time, based on systematic review. Gastroenterology. 142:46-54, e42, quiz e30.

4. Henderson P, van Limbergen JE, Schwarze J, Wilson DC. (2011) Function of the intestinal epithelium and its dysregulation in inflammatory bowel disease. Inflamm. Bowel Dis. 17:382-95.

5. Ananthakrishnan AN. (2015) Epidemiology and risk factors for IBD. Nat. Rev. Gastroenterol. Hepatol. 12:205-17.

6. North BJ, Verdin E. (2004) Sirtuins: Sir2-related NAD-dependent protein deacetylases. Genome Biol. 5:224

7. Caruso R, et al. (2014) Defective expression of SIRT1 contributes to sustain inflammatory pathways in the gut. Mucosal Immunol. 7:1467-79.

8. Yang H, et al. (2012) SIRT1 activators suppress inflammatory responses through promotion of p65 deacetylation and inhibition of NF-kappaB activity. PLoS One. 7:e46364.

9. Kugel S, Mostoslavsky R. (2014) Chromatin and beyond: the multitasking roles for SIRT6. Trends Biochem. Sci. 39:72-81.

10. Kanfi Y, Naiman S, Amir G, Peshti V, Zinman G. (2012) The sirtuin SIRT6 regulates lifespan in male mice. Nature. 483:218-21.

11. Mostoslavsky R, et al. (2006) Genomic instability and aging-like phenotype in the absence of mammalian SIRT6. Cell. 124:315-29.

12. el Marjou F, et al. (2004) Tissue-specific and inducible Cre-mediated recombination in the gut epithelium. Genesis. 39:186-93.

13. Kim HS, et al. (2010) Hepatic-specific disruption of SIRT6 in mice results in fatty liver formation due to enhanced glycolysis and triglyceride synthesis. Cell Metab. 12:224-36.

14. Chogle A, et al. (2011) Milk fat globule-EGF factor 8 is a critical protein for healing of dextran sodium sulfate-induced acute colitis in mice. Mol. Med. 17:502-07. 
15. Bu HF, et al. (2007) Milk fat globule-EGF factor $8 /$ lactadherin plays a crucial role in maintenance and repair of murine intestinal epithelium. J. Clin. Invest. 117:3673-83.

16. Zhu YQ, Tan XD. (2005) TFF3 modulates NF-\{kappa $\} \mathrm{B}$ and a novel negative regulatory molecule of NF-\{kappa\}B in intestinal epithelial cells via a mechanism distinct from TNF-\{alpha\}. Am. J. Physiol. Cell Physiol. 289:C1085-93.

17. Whitehead RH, VanEeden PE, Noble MD, Ataliotis P, Jat PS. (1993) Establishment of conditionally immortalized epithelial cell lines from both colon and small intestine of adult $\mathrm{H}$-2Kb-tsA58 transgenic mice. Proc. Natl. Acad. Sci. USA. 90:587-91.

18. Yan F, et al. (2013) A Lactobacillus rhamnosus GG-derived soluble protein, p40, stimulates ligand release from intestinal epithelial cells to transactivate epidermal growth TNFfactor receptor. J. Biol. Chem. 288:30742-51.

19. Wang X, Bu HF, Liu SX, De Plaen IG, Tan XD (2015) Molecular mechanisms underlying the regulation of the MFG-E8 gene promoter activity in physiological and inflammatory conditions. J. Cell. Biochem. 116:1867-79.

20. Viennois E, Chen F, Laroui H, Baker MT, Merlin D. (2013) Dextran sodium sulfate inhibits the activities of both polymerase and reverse transcriptase: lithium chloride purification, a rapid and efficient technique to purify RNA. BMC Res. Notes. 6:360.

21. Livak KJ, Schmittgen TD. (2001) Analysis of relative gene expression data using real-time quantitative PCR and the 2(-Delta Delta C(T)) Method. Methods. 25:402-08.

22. Bu HF, et al. (2006) Lysozyme-modified probiotic components protect rats against polymicrobial sepsis: role of macrophages and cathelicidin-related innate immunity. J. Immunol. 177:8767-76.

23. Nunes T, Bernardazzi C, de Souza HS. (2014) Cell death and inflammatory bowel diseases: apoptosis, necrosis, and autophagy in the intestinal epithelium. Biomed. Res. Int. 2014:218493.

24. Negroni A, Cucchiara S, Stronati L. (2015) Apoptosis, necrosis, and necroptosis in the gut and intestinal homeostasis. Mediators Inflamm. 2015:250762.

25. Subramanian A, et al. (2005) Gene set enrichment analysis: a knowledge-based approach for interpreting genome-wide expression profiles. Proc. Natl. Acad. Sci. USA. 102:15545-50.

26. Mootha VK, et al. (2003) PGC-1alpha-responsive genes involved in oxidative phosphorylation are coordinately downregulated in human diabetes. Nat. Genet. 34:267-73.

27. Supek F, Bosnjak M, Skunca N, Smuc T. (2011) REVIGO summarizes and visualizes long lists of gene ontology terms. PLoS One. 6: e21800.

28. Neurath MF. (2014) Cytokines in inflammatory bowel disease. Nat. Rev. Immunol. 14:329-42.

29. Ito R, et al. (2006) Interferon-gamma is causatively involved in experimental inflammatory bowel disease in mice. Clin. Exp. Immunol. 146:330-38
30. Zhong L, Mostoslavsky R. (2010) SIRT6: a master epigenetic gatekeeper of glucose metabolism. Transcription. 1:17-21.

31. Kawahara TL, et al. (2011) Dynamic chromatin localization of Sirt6 shapes stress- and aging-related transcriptional networks. PLoS Genet. 7:e1002153.

32. Lerrer B, Gertler AA, Cohen HY. (2016) The complex role of SIRT6 in carcinogenesis. Carcinogenesis. 37:108-18.

33. Kim KA, et al. (2008) R-Spondin family members regulate the Wnt pathway by a common mechanism. Mol. Biol. Cell. 19:2588-96.

34. Binnerts ME, et al. (2007) R-Spondin1 regulates Wnt signaling by inhibiting internalization of LRP6. Proc. Natl. Acad. Sci. USA. 104:14700-05.

35. Zhao J, et al. (2007) R-spondin1, a novel intestinotrophic mitogen, ameliorates experimental colitis in mice. Gastroenterology. 132:1331-43.

36. Kim KA, et al. (2005) Mitogenic influence of human R-spondin1 on the intestinal epithelium. Science. 309:1256-59.

37. Lim R, Barker G, Lappas M. (2013) SIRT6 is decreased with preterm labor and regulates key terminal effector pathways of human labor in fetal membranes. Biol. Reprod. 88:17.

38. Balestrieri ML, et al. (2015) Sirtuin 6 expression and inflammatory activity in diabetic atherosclerotic plaques: effects of incretin treatment. Diabetes. 64:1395-406.

39. Liu R, Liu H, Ha Y, Tilton RG, Zhang W. (2014) Oxidative stress induces endothelial cell senescence via downregulation of Sirt6. Biomed. Res. Int. 2014:902842.

40. Liszt G, Ford E, Kurtev M, Guarente L. (2005) Mouse Sir2 homolog SIRT6 is a nuclear ADPribosyltransferase. J. Biol. Chem. 280:21313-20.

41. Corda D, Di Girolamo M. (2003) Functional aspects of protein mono-ADP-ribosylation. EMBO J. 22:1953-58

42. Yang B, Zwaans BM, Eckersdorff M, Lombard DB. (2009) The sirtuin SIRT6 deacetylates H3 K56Ac in vivo to promote genomic stability. Cell Cycle. 8:2662-63.

43. Sebastian C, et al. (2012) The histone deacetylase SIRT6 is a tumor suppressor that controls cancer metabolism. Cell. 151:1185-99.

44. Michishita E, et al. (2008) SIRT6 is a histone H3 lysine 9 deacetylase that modulates telomeric chromatin. Nature. 452:492-96.

45. Michishita E, et al. (2009) Cell cycle-dependent deacetylation of telomeric histone H3 lysine K56 by human SIRT6. Cell Cycle. 8:2664-66.

46. Kawahara TL, et al. (2009) SIRT6 links histone H3 lysine 9 deacetylation to NF-kappaB-dependent gene expression and organismal life span. Cell. 136:62-74.

47. Jiang H, et al. (2013) SIRT6 regulates TNF-alpha secretion through hydrolysis of long-chain fatty acyl lysine. Nature. 496:110-13.

48. Bhanja P, et al. (2009) Protective role of R-spondin1, an intestinal stem cell growth factor, against radiation-induced gastrointestinal syndrome in mice. PLoS One. 4:e8014.
49. Zhao J, Kim KA, Abo A. (2009) Tipping the balance: modulating the Wnt pathway for tissue repair. Trends Biotechnol. 27:131-36.

50. Zhao J, et al. (2009) R-Spondin1 protects mice from chemotherapy or radiation-induced oral mucositis through the canonical Wnt/betacatenin pathway. Proc. Natl. Acad. Sci. USA. 106:2331-36

Cite this article as: Liu F, et al. (2017) Sirtuin-6 preserves R-spondin-1 expression and increases resistance of intestinal epithelium to injury in mice. Mol. Med. 23:272-284. 